\title{
Design and Development of ArF Photoresist for Implant Layers
}

\author{
Youngsoo Yang, Jun Ho Lee, Tae Ho Kim, Seung Jib Choi, Sang Jun Choi, Tuwon Chang, \\ Seongjune Kim*, Dongwon Kim*, Hyereun Kim*, Youngho Kim*, \\ Sung-Ki Chae* and Jae Hyun Kim** \\ Polymer Synthesis Team, Electronic Materials Research Center, \\ Samsung Cheil Industries, Inc., 332-2 Gocheon-Dong, Uiwang-Si, Gyeonggi-Do, Korea \\ *Manufacturing Technology Team, Infra Technology Service Center, \\ Samsung Electronics, San \#16 Banwol-Dong, Hwasung-City, Gyeonggi-Do, Korea \\ **hawk_kim@samsung.com
}

\begin{abstract}
Our recent progress on the development of ArF photoresist (PR) for implant layers is reported. Since scum-free patterning is the critical property of an implant PR, a specific focus has been on eliminating scum while maintaining other lithographic performances. Through the optimization of formulation with respect to $\mathrm{k}$ value and the use of novel monomers with less bulky substituents, a poly-methacrylate implant PR with minimal scum was obtained. Finally, ion-stopping ability of the prepared PR was tested via secondary ion mass spectrometry (SIMS) and was found to be sufficient for implant layer applications.
\end{abstract}

Keyword: photoresist, $193 \mathrm{~nm}$ lithography, scum, ion implantation.

\section{Introduction}

Advances in semiconductor industry have put increasing demands on photolithography to produce smaller patterns. Applying irradiation sources of increasingly smaller wavelengths has been a major approach to meet them. Applications of improved exposure technologies, utilizing g-line $(436 \mathrm{~nm})$, i-line $(365 \mathrm{~nm}), \operatorname{KrF}(248 \mathrm{~nm})$, and $\operatorname{ArF}(193 \mathrm{~nm})$, have been accompanied by developments of suitable photoresists (PR's) for respective systems, such as novolak/ diazonaphthoquinone, poly-hydroxystyrene, and poly-methacrylate [1-4]. Although a PR is generally classified by the corresponding exposure source, it can also be categorized according to its applied layer.

Current integrated circuit manufacturing can require more than ten ion implantation steps and applicable PR's [5]. Ion implantation introduces dopants such as boron, phosphorus, or arsenic atoms into the silicon wafer. Each dopant atom creates a charge carrier in the substrate, either a hole or an electron, modifying its conductivity.

PR for an implant layer is unique in that it directly adheres to the substrate without the organic bottom anti-reflective coating (BARC). Photolithography of an implant layer do not use the BARC because the dry etch process to remove it may damage the substrate subsequently to be implanted. Considering the above, desired properties of an implant PR include (1) scum-free patterning to ensure even ion implantation, (2) excellent adhesion strength to avoid pattern lifting, (3) good masking ability of the unexposed area against the ion implantation, and (4) resistance to materialization of standing waves.

Currently, i-line and $\mathrm{KrF}$ photolithographies have predominantly been used for implant layers. However, as the demand to produce denser integrated circuits continues, it is inevitable that 
the need for $193 \mathrm{~nm}$ photolithography for implant layers will arise. Hence, we set out to develop a poly-methacrylate based ArF PR for implant layers that satisfies aforementioned properties.

The most commonly used ArF PR's utilize poly-methacrylates because of their transparency at $193 \mathrm{~nm}$ and bulky alicyclic substituents on them to maximize the dry etch resistance [6]. However, bulky alicyclic substituents increase the PR's hydrophobicity, presumably undermining desired properties for implant layer applications, such as scum-free patterning and high adhesion strength.

We envisioned that through optimizing formulation and employing novel methacrylate monomers, a poly-methacrylate PR capable of producing scum-free patterns with sufficient adhesion strength could be obtained. In this report, we present our recent efforts in developing an $\mathrm{ArF}$ PR for implant layers.

\section{Method}

Each PR consisted of poly-methacrylate, photoacid generator (PAG), quencher, additives, and solvent. Standard polymer (Polymer-A) was prepared with 2-methyl-2-adamantyl methacrylate (MAdMA), $\alpha$-hydroxy- $\gamma$-butyrolactone methacrylate ( $\alpha$-GBLMA), and hydroxy-1methacryloxyadamantane (HAdMA) as monomers. Novel monomers ( $\mathrm{M}-\mathrm{a}$ and $\mathrm{M}-\mathrm{b}$ ) were incorporated to prepare other polymers (Polymer-B, Polymer-C, and Polymer-D) as described in Table 1. All four polymers were prepared to have the same molar ratios of three monomers and negligible differences in molecular weights (Mw) and polydispersity indexes (PDI).

PAG's with various transparencies were used. These contained cations, which determined $\mathrm{k}$ values, and anions, which turned into acids upon irradiation and diffused during the post exposure bake (PEB). A piperidine based quencher was used. A 7:3 mixture of propylene glycol methylether acetate and cyclohexanone was used as the solvent.

Prepared PR's were coated onto $200 \mathrm{~mm}$ wafers with described thicknesses. The coated films were baked (SB: Soft Bake) at $110{ }^{\circ} \mathrm{C}$ for $60 \mathrm{~s}$, exposed, re-baked (PEB) at $110{ }^{\circ} \mathrm{C}$ for $60 \mathrm{~s}$, and then developed with an aqueous $0.26 \mathrm{~N}$ tetramethylammonium hydroxide (TMAH) solution via puddle method. Lithography experiments were carried out with Nikon NSR-S308F with 0.85 numerical aperture and conventional illumination. Scanning electron micrographs (SEM) were measured with Hitachi S-9260. Patterns examined for the study were $150 \mathrm{~nm}$ iso-spaces.

Ion implantation experiments were performed with Varian VIISta80-HP, using boron fluoride with $8 \mathrm{keV}$ impact energy and $5 \times 10^{13}$ boron $/ \mathrm{cm}^{2}$ dose. Wafers were then stripped of PR films with PSK DAS2000 via $\mathrm{O}_{2}$ ashing, followed by subsequent cleanings with Hitachi NXWET and secondary ion mass spectrometry (SIMS) measurements. The instrument used for the SIMS was CAMECA IMS-6f Magnetic Sector SIMS with $\mathrm{O}_{2}{ }^{+}$Gun with $7.5 \mathrm{keV}$ impact energy and 300 nA current.

\section{Results and Discussion}

3.1. Effect of $\mathrm{k}$ value on lithographic performance and scum.

In general, thicknesses of implant PR films are much higher than those of normal PR films in order to prevent ion penetrations. Consequently, transparency of an implant PR film plays a crucial role in scum formation since it dictates how much of the irradiation can reach the bottom. In a poly-methacrylate PR, the type and the amount of PAG significantly affect film transparency. Through PAG optimization using a conventional ArF PR polymer (Polymer-A), the appropriate transparency, expressed as $\mathrm{k}$,

Table 1. Prepared Polymers

\begin{tabular}{|c|c|c|c|c|c|c|}
\hline \multirow{2}{*}{ Name } & \multicolumn{3}{|c|}{ Monomers } & \multirow{2}{*}{$\mathrm{T}_{\mathrm{g}}\left({ }^{\circ} \mathrm{C}\right)$} & \multirow{2}{*}{$\mathrm{Mw}$} & \multirow{2}{*}{ PDI } \\
\hline & $40 \mathrm{~mol} \%$ & $30 \mathrm{~mol} \%$ & $30 \mathrm{~mol} \%$ & & & \\
\hline Polymer-A & MAdMA & $\alpha$-GBLMA & HAdMA & 197.2 & $9.9 \mathrm{k}$ & 1.53 \\
\hline Polymer-B & M-a & $\alpha-$ GBLMA & HAdMA & 169.5 & $10.5 \mathrm{k}$ & 1.76 \\
\hline Polymer-C & MAdMA & $\alpha-$ GBLMA & M-b & 168.3 & $10.0 \mathrm{k}$ & 1.48 \\
\hline Polymer-D & M-a & $\alpha-$ GBLMA & $M-b$ & 145.2 & $9.6 \mathrm{k}$ & 1.59 \\
\hline
\end{tabular}


was found. PAG's used in this study contained identical anions to keep the acid diffusion length constant.

PR-1, PR-2, and PR-3 were prepared with Polymer-A and same total amounts but varying ratios of PAG mixtures (Table 2).

Table 2. Photoresists prepared with Polymer-A and their $\mathrm{k}$ values

\begin{tabular}{|c|c|}
\hline Name & $\mathrm{k}$ \\
\hline PR-1 & 0.0394 \\
\hline PR-2 & 0.0386 \\
\hline PR-3 & 0.0291 \\
\hline
\end{tabular}

When lithographic performances of these PR's were evaluated for $150 \mathrm{~nm}$ iso-space patterning at $4000 \AA$ thickness, clean scum-free patterns were obtained for PR-3 across a wide exposure dose range while PR-1 and PR-2 showed limited ranges of or no exposure dose margin due to scum (Figure 1). Since the PR films with a higher $\mathrm{k}$ values absorbed light more effectively, smaller amount of it could reach the bottom, causing less acid generated close to the substrate. This disparity in the acid amounts led to differences in the extent of the deprotection reaction. Greater amount of unreacted protecting groups caused the incomplete dissolution of the PR by the developer, leaving scum. In this experiment with Polymer-A, k value smaller than 0.03 was needed to minimize this disparity and achieve sufficiently scum-free lithographic performance at $4000 \AA$ thickness, and subsequent experiments were performed using the formulation of PR-3.

3.2. Effect of incorporating $\mathrm{M}-\mathrm{a}$ and $\mathrm{M}-\mathrm{b}$ on lithographic performance and scum.

While PR-3 successfully yielded scum-free 150 $\mathrm{nm}$ iso-space patterns at $4000 \AA$ thickness, it failed to prevent scum formation at $5500 \AA$ thickness. To overcome this limitation, monomers in Polymer-A were replaced with other monomers bearing similar functions. $\mathrm{M}-\mathrm{a}$ and $\mathrm{M}-\mathrm{b}$, were used to substitute MAdMA and HAdMA, respectively (Table 1).

Monomer M-a contained a relatively less bulky acid labile substituent while monomer $\mathrm{M}-\mathrm{b}$ contained a relatively less bulky alicycle.
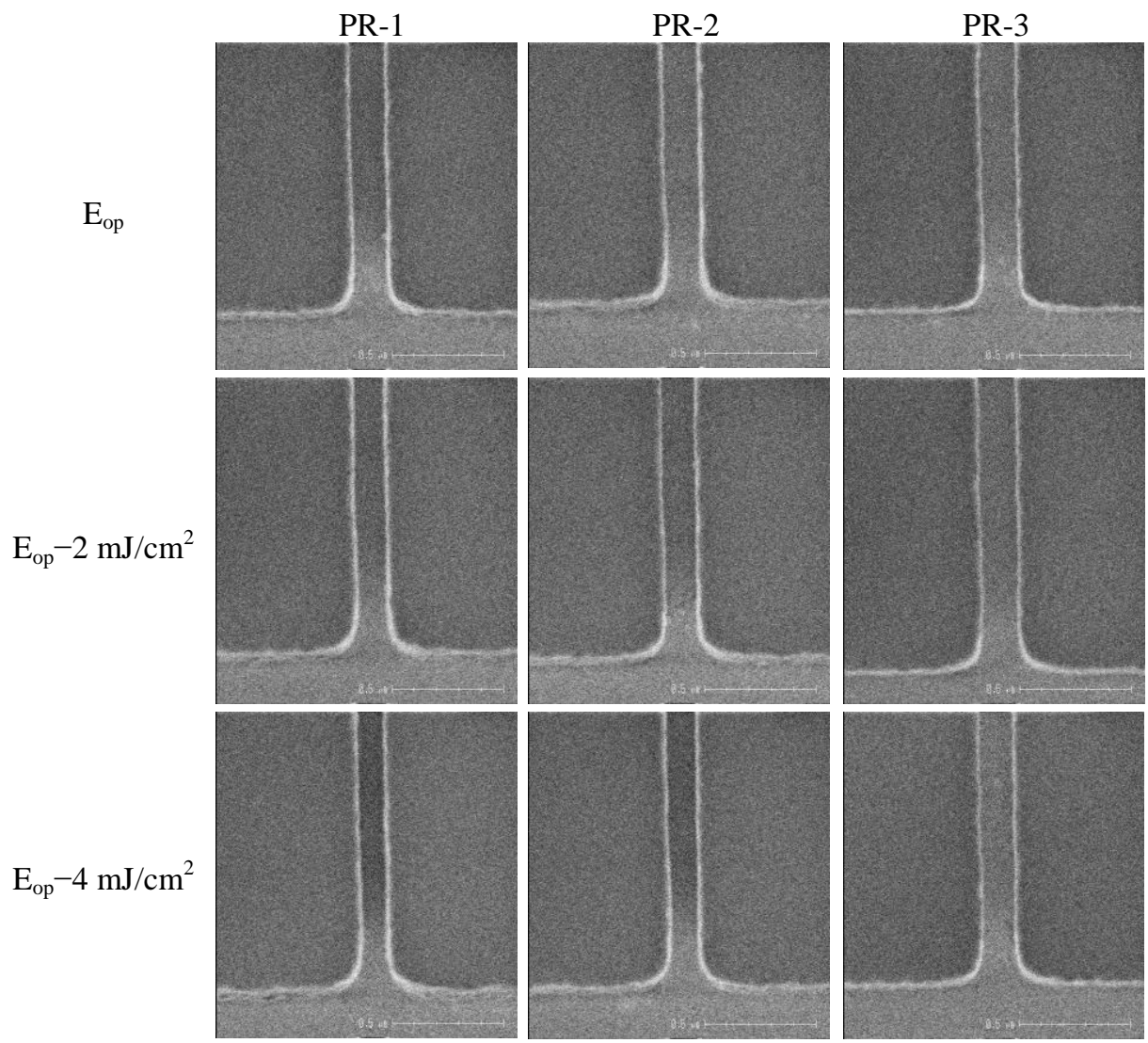

Figure 1. Top-down SEM images of PR-1, PR-2, and PR-3 at $E_{o p}, E_{o p}-2 \mathrm{~mJ} / \mathrm{cm}^{2}$, and $E_{o p}-4 \mathrm{~mJ} / \mathrm{cm}^{2}(150 \mathrm{~nm}$ iso-space; $4000 \AA$ ) 
Although $\alpha$-GBLMA was not bulky, its replacement with an alternative monomer was still examined. However, all attempts to substitute $\alpha$-GBLMA failed to yield an applicable PR, suggesting the essentiality of the lactone functionality in a poly-methacrylate PR [7].

The optimized PAG system from section 3.1 was employed to prepare three additional PR's, PR-4, PR-5, and PR-6 from Polymer-B, Polymer-C, and Polymer-D, respectively. Presented in Figure 2 are $150 \mathrm{~nm}$ iso-space lithographic images of these PR's along with PR-3 at 5500 Å thickness.

While PR-3 could not produce $150 \mathrm{~nm}$ iso-space patterns without scum, PR-4 and PR-5, which respectively replaced only MAdMA and only HAdMA with less bulky M-a and M-b, could produce scum-free patterns at optimal exposure doses $\left(E_{o p}\right)$. However, decrease in the exposure dose by $2 \mathrm{~mJ} / \mathrm{cm}^{2}$ or $4 \mathrm{~mJ} / \mathrm{cm}^{2}$ readily led to scum formation. PR-6, which replaced both MAdMA and HAdMA, provided clean patterns not only at the $E_{o p}$ but also at lower doses. Further experiments showed that PR-4 and PR-5 could produce scum-free $130 \mathrm{~nm}$ iso-space patterns with minimal depth of focus (DOF) margins, while
PR-6 exhibited good lithographic performances even at $110 \mathrm{~nm}$ resolutions with a good DOF margin.

This result was rationalized in terms of hydrophilicity and glass transition temperature $\left(\mathrm{T}_{\mathrm{g}}\right)$ of the polymer. As less bulky monomers were introduced into the polymer, the PR's hydrophilicity increased, enhancing its overall solubility by the basic developer. As a result, dissolution property of the PR's exposed area also improved. On the other hand, incorporations of the less bulky monomers, $\mathrm{M}-\mathrm{a}$ and $\mathrm{M}-\mathrm{b}$, decreased $\mathrm{T}_{\mathrm{g}}$ of the polymer as shown in Table 1 . The polymer matrix with a lower $\mathrm{T}_{\mathrm{g}}$ allowed for the acid diffusion and the deprotection reaction to occur more effectively during PEB, increasing the dissolution property of the PR's exposed area, which ultimately manifested into less scum formation.

Another critical property of an implant PR is high adhesion strength to avoid pattern lifting. A poly-hydroxystyrene based KrF PR generally has higher adhesion strength than a poly-methacrylate based ArF PR due to abundant phenolic functionalities in the former. We predicted that using a poly-methacrylate with less bulky

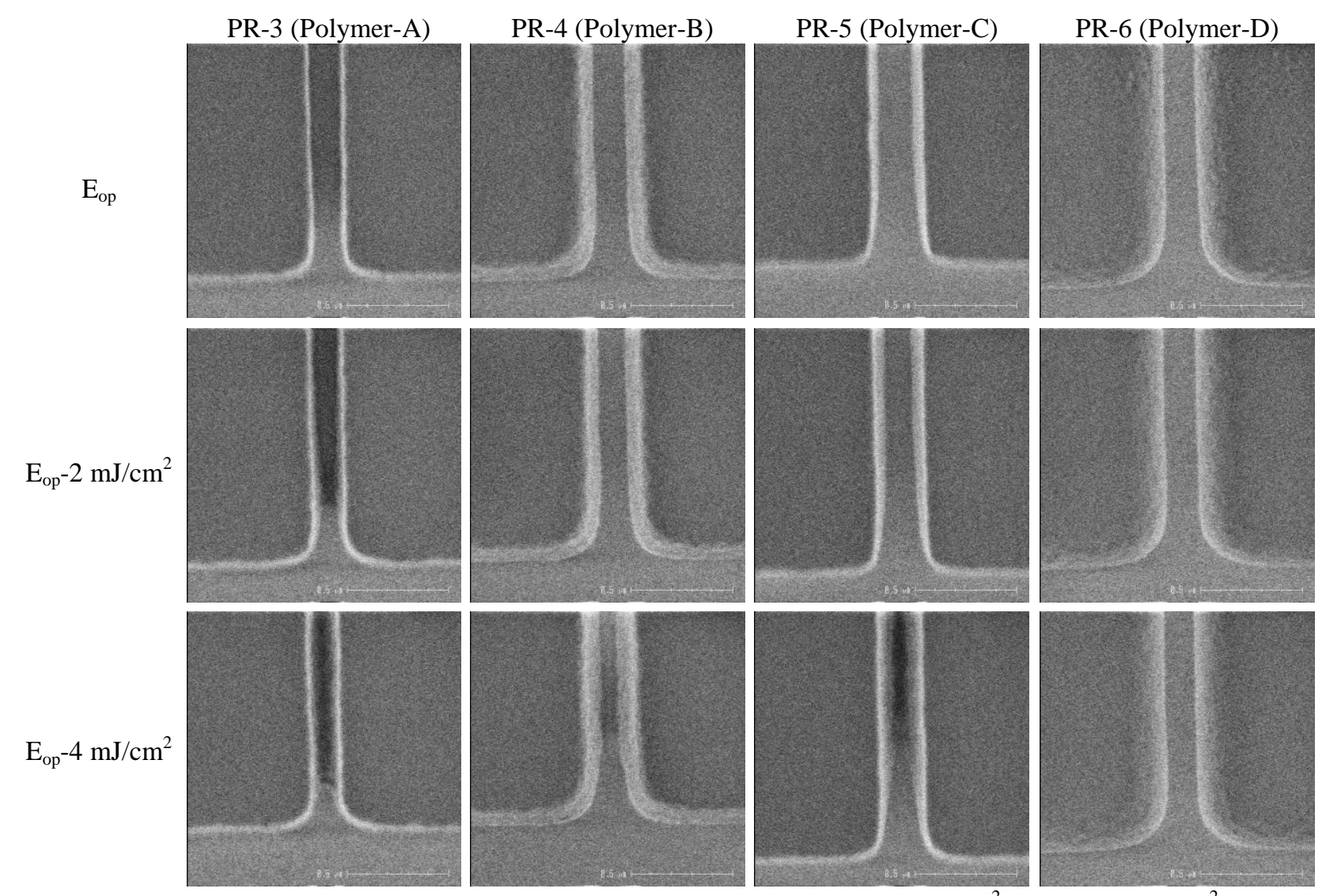

Figure 2. Top-down SEM images of PR-3, PR-4, PR-5, and PR-6 at $E_{o p}, E_{o p}-2 \mathrm{~mJ} / \mathrm{cm}^{2}$, and $E_{o p}-4 \mathrm{~mJ} / \mathrm{cm}^{2}(150 \mathrm{~nm}$ iso-space; $5500 \AA$ ) 


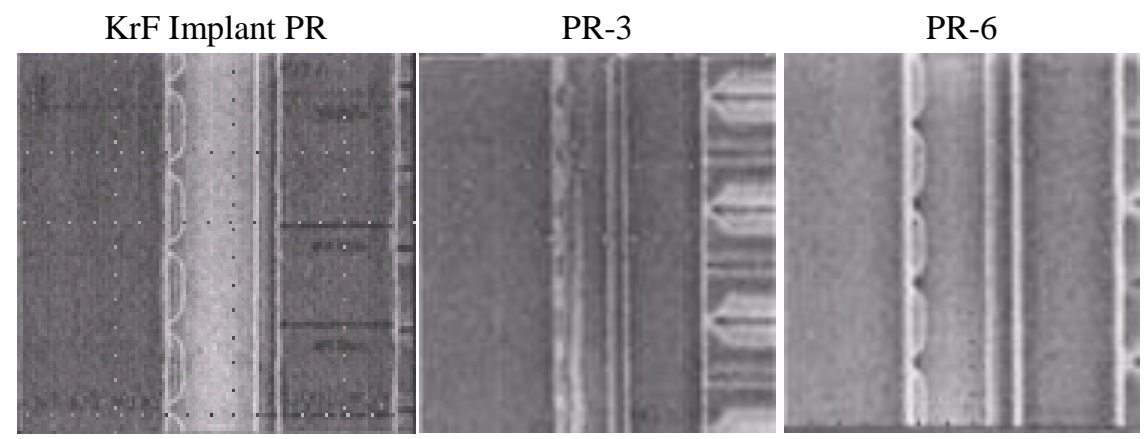

Figure 3. Top-down SEM images of KrF Implant PR, PR-3, and PR-6 at the weak point of an implant layer

substituents would increase hydrophilicity, rendering high adhesion strength.

To test the effect of the substituent size on PR's adhesion strength, PR-3, PR-6, and a KrF implant $\mathrm{PR}$ were subjected to produce more demanding patterns. As shown in Figure 3, PR-3 could not produce the fine patterns due to pattern lifting while $\mathrm{KrF}$ implant $\mathrm{PR}$ with a $248 \mathrm{~nm}$ exposure tool could produce the pattern. Much to our delight, comparable patterns were achieved with PR-6.

\subsection{Masking ability}

Effective shielding of the substrate during ion implantation is another essential property of an implant PR. Therefore, masking ability of PR- 6 was examined. PR films of varying thicknesses were coated onto p-type wafers. After boron fluoride implantation and PR stripping, boron concentrations at different sputter depths were measured via SIMS to determine the penetration of boron ions (Figure 4).

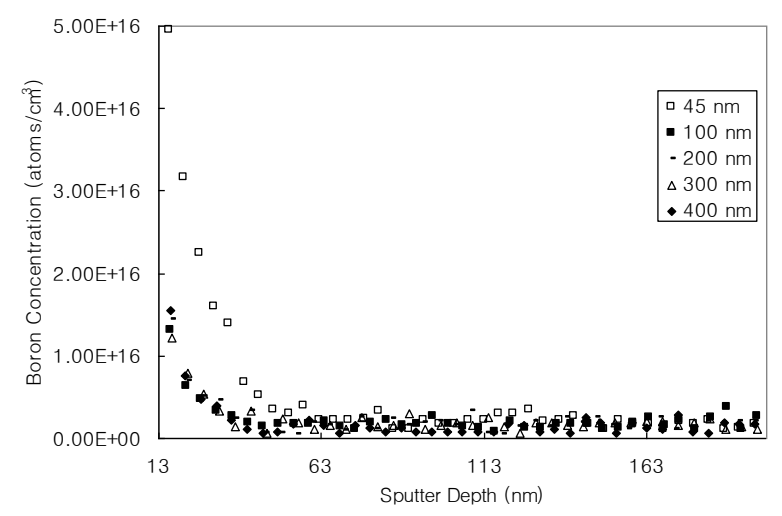

Figure 4. Boron concentration (atoms $/ \mathrm{cm}^{3}$ ) vs. sputter depth (nm), using various thicknesses of PR-6 as masks

Despite the ubiquitous basal level of boron detections in all p-type wafers, a clear difference between the result using $45 \mathrm{~nm}$ thickness and all other thicknesses was observed. It could be inferred that the basal boron detection level in all wafers originated from wafers themselves, and boron ions from implantation could not penetrate PR films with thicknesses greater than $45 \mathrm{~nm}$.

\section{Conclusion}

A poly-methacrylate based ArF PR for implant layers was designed and developed. Since absence of PR scum is the critical characteristic of an implant PR, formulation was first optimized to minimize scum formation. It was found that PR's with lower $\mathrm{k}$ values favored complete dissolution of the exposed areas since the irradiation could reach the bottom without much loss of intensity.

Employing novel monomers with less bulky substituents further enhanced the scum-free patterning property by providing polymers with higher hydrophilicities and lower $\mathrm{T}_{\mathrm{g}}$ 's. Higher hydrophilicity led to better solubility of the PR by the basic developer, and lower $T_{g}$ favored the acid diffusion and the deprotection reaction. Application of these monomers also improved the prepared PR's robustness against pattern lifting. Finally, ion stopping ability of this PR was tested via SIMS to demonstrate the complete blockings of boron ions for all film thicknesses above $100 \mathrm{~nm}$.

Thus, presented are our efforts in developing a poly-methacrylate ArF PR for implant layers. Further works to increase the PR thickness without loss of lithographic performance and to improve resolution at more complex patterns are underway.

\section{References}

1. O. Süss, Liebigs Ann. Chem., 556 (1944), 65. 
2. H. Ito, C. G. Willson, and J. M. J. Fréchet, (1982), Digest of Technical Papers of 1982 Symposium on VLSI Technology, 86.

3. H. Ito and C. G. Willson, "Polymers in Electronics", T. Davidson, Eds. Washington, D. C., p. 11.

4. R. R. Kunz, D. Allen, W. D. Hinsberg, and G. M. Wallraff, Proc. SPIE, 1925 (1993), 167.
5. M. Ross and W. R. Livesay, Proc. SPIE, 2724 (1996), 632.

6. H. G. Craighead, J. C. White, R. E. Howard, L. D. Jackel, R. E. Behringer, J. E. Sweeney, and R. W. Epworth, J. Vac. Sci. Technol. B, 1 (1983), 1186.

7. H. Ito, H. D. Truong, and P. J. Brock, Proc. SPIE, 6923 (2008), 692318. 